Homology, Homotopy and Applications, vol.11(2), 2009, pp.189-194

\title{
COMMUTATIVE $\Gamma$-RINGS DO NOT MODEL ALL COMMUTATIVE RING SPECTRA
}

\author{
TYLER LAWSON
}

\author{
(communicated by Brooke Shipley)
}

\begin{abstract}
We show that the free $E_{\infty}$-algebra on a zero-cell cannot be modeled by a commutative $\Gamma$-ring. The proof shows that DyerLashof operations of positive degree must vanish on the zero'th homology of such an object.
\end{abstract}

\section{Introduction}

There are now several peacefully coexisting models for the stable homotopy category that admit amenable symmetric monoidal structures, and this has led to a great deal of new activity in the subject. The most commonly used models are $\mathbb{S}$-modules [3], symmetric spectra [4], orthogonal spectra [7], and $\Gamma$-spaces $[\mathbf{9}]$ with a smash product introduced by Lydakis [6].

Each of these categories has its advantages and its quirks. The main distinction between $\Gamma$-spaces and the other major models of homotopy theory is that $\Gamma$-spaces only model connective spectra. On the other hand, they have the advantage that they are simple to define, as well as being closely tied to common infinite loop space machines, to algebraic $K$-theory, and to Goodwillie calculus.

The symmetric monoidal structure on $\Gamma$-spaces gives rise to a notion of a commutative $\Gamma$-ring. There is a natural realization functor from these objects to symmetric spectra that is symmetric monoidal, and hence takes commutative $\Gamma$-rings to commutative symmetric ring spectra [7].

The notions of commutative ring objects in $\mathbb{S}$-modules, symmetric spectra, and orthogonal spectra can be made homotopically meaningful and equivalent. However, as mentioned in the introduction of [8], it seems unlikely that many such "structured commutative ring spectra," or $E_{\infty}$-algebras, can be modeled by a commutative $\Gamma$-ring.

The purpose of this note is to show that Dyer-Lashof operations of positive degree all vanish on the zero'th homology of a spectrum associated to a commutative $\Gamma$-ring. Therefore, not all connective commutative ring spectra can be modeled. The canonical example is, as one might guess, the free algebra on a zero-cell. Commutative $\Gamma$-rings then exist as some intermediate category between simplicial commutative rings and connective $E_{\infty}$-algebras.

Partially supported by NSF grant 0805833.

Received May 26, 2009, revised June 26, 2009; published on November 13, 2009.

2000 Mathematics Subject Classification: 55P43, 55S12, 55P42.

Key words and phrases: Gamma-space, ring spectrum, Dyer-Lashof operation

This article is available at http://intlpress.com/HHA/v11/n2/a9

Copyright (C) 2009, International Press. Permission to copy for private use granted. 


\section{Proof of main result}

We recall some definitions and results from $[\mathbf{1}, \mathbf{8}]$. Let $\Gamma^{o}$ be (a skeletal replacement for) the category of finite based sets, and $\mathcal{S}$ the category of based simplicial sets ("spaces"). We let $S^{n}=\left(S^{1}\right)^{\wedge n}$ be the $n$-fold smash product of the simplicial circle.

Definition 2.1. A $\Gamma$-space is a functor $X: \Gamma^{o} \rightarrow \mathcal{S}$ such that $X(*)=*$.

A $\Gamma$-space prolongs to a functor on all based spaces as follows: For an arbitrary based set $S$, define

$$
X(S)=\underset{T \subset S,|T|<\infty}{\operatorname{colim}} X(T)
$$

and for a based space $K=\left\{K_{n}\right\}$, we let $X(K)$ be the realization (diagonal) of the simplicial space $\left\{X\left(K_{n}\right)\right\}$. Weak equivalences between spaces give rise to weak equivalences between values of $X$ [6, Prop. 5.20].

For based sets $S$ and $T$, there is a natural "assembly map"

$$
S \wedge X(T) \cong \bigvee X(T) \rightarrow X(\bigvee T) \cong X(S \wedge T)
$$

which, by levelwise application, prolongs to an assembly map

$$
K \wedge X(L) \rightarrow X(K \wedge L)
$$

This is natural in based spaces $K$ and $L$ and is an isomorphism if $K=*$ or $K=S^{0}$. We can make the following definition.

Definition 2.2. The associated spectrum $\mathcal{S} p(X)$ is the symmetric spectrum $\left\{X\left(S^{n}\right)\right\}$, with structure maps given by the assembly maps

$$
S^{m} \wedge X\left(S^{n}\right) \rightarrow X\left(S^{m} \wedge S^{n}\right) .
$$

This functor is a composite of functors denoted by $\mathbb{P}$ in $[\mathbf{7}]$. The symmetric spectrum $\mathcal{S} p(X)$ is always connective and has semistable homotopy groups, meaning that the derived homotopy group $\pi_{k}$ of homotopy classes of maps $S^{k} \rightarrow \mathcal{S} p(X)$ can be calculated as colim $\pi_{n+k} X\left(S^{n}\right)$. (In a version based on topological spaces, the functor $\mathcal{S} p$ has this property because it factors through orthogonal spectra.)

Lemma 2.3. The image of the set $\pi_{0}\left(X\left(S^{0}\right)\right)$ generates the group $\pi_{0}(\mathcal{S} p(X))$.

Proof. It suffices to show that the suspension maps $S^{n} \wedge X\left(S^{0}\right) \rightarrow X\left(S^{n}\right)$ are surjective on $\pi_{n}$. There is a "collapse" weak equivalence of simplicial sets $S^{n} \rightarrow \tilde{S}^{n}$ from the $n$-fold smash product to a complex with only two non-degenerate simplices, in degrees 0 and $n$. In the commutative diagram

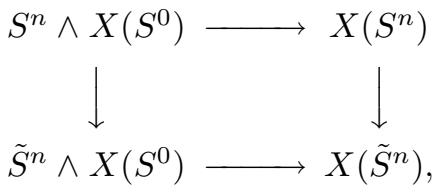

the vertical maps are weak equivalences, and so it suffices to show that the lower assembly map is surjective on $\pi_{n}$. 
However, as a map of simplicial spaces the assembly map in the lower row is the $\operatorname{map}\left(\tilde{S}^{n}\right)_{k} \wedge X\left(S^{0}\right) \rightarrow X\left(\left(\tilde{S}^{n}\right)_{k}\right)$, which is an isomorphism in degrees $n$ and lower. The resulting map of geometric realizations is therefore an isomorphism on $n$-skeleta, and hence $n$-connected.

There is a symmetric monoidal structure $\wedge$ on $\Gamma$-spaces, based on left Kan extension, such that a map $X \wedge Y \rightarrow Z$ is equivalent to a natural family of maps

$$
X(S) \wedge Y(T) \rightarrow Z(S \wedge T)
$$

for finite based sets $S$ and $T$. The smash product has as unit the identity functor $\mathbb{S}(S)=S$.

Definition 2.4. A commutative $\Gamma$-ring is a $\Gamma$-space $R$ with maps $R \wedge R \rightarrow R$ and $\mathbb{S} \rightarrow R$ making $R$ a commutative, associative monoid under $\wedge$.

In particular, a commutative $\Gamma$-ring $R$ has natural multiplication maps

$$
R(X) \wedge R(Y) \rightarrow R(X \wedge Y)
$$

commuting with the twist isomorphism, and has a natural commutative monoid structure on $R\left(S^{0}\right)$. The functor $\mathcal{S} p$ is lax symmetric monoidal, and so the associated symmetric spectrum $\mathcal{S} p(R)$ is then naturally a commutative ring object. Explicitly, the maps

$$
R\left(S^{n}\right) \wedge R\left(S^{m}\right) \rightarrow R\left(S^{n} \wedge S^{m}\right)
$$

obtained by applying the multiplication maps levelwise to the simplicial set $S^{n} \wedge$ $S^{m}$, give the symmetric spectrum a commutative ring structure, and $R\left(S^{0}\right)$ is a commutative topological monoid. (As in the objection of Lewis [5], the fact that the zero'th space of a commutative symmetric ring spectrum is too commutative is an essential reason why the positive stable model structure on symmetric spectra $[\mathbf{7}, \S 14]$ is necessary to obtain a good model category of commutative ring objects.)

For a $\Gamma$-space $X$ and a based space $K$, we have an object $X \wedge K$ given by

$$
(X \wedge K)(S)=X(S) \wedge K
$$

The functor $\mathbb{S} \wedge(-)$ is part of the adjunction

$$
\operatorname{Map}_{\mathcal{S}}\left(K, X\left(S^{0}\right)\right) \cong \operatorname{Map}_{\Gamma}(\mathbb{S} \wedge K, X),
$$

and therefore this functor preserves colimits. There are natural isomorphisms

$$
(\mathbb{S} \wedge K) \wedge(\mathbb{S} \wedge L) \cong \mathbb{S} \wedge(K \wedge L)
$$

making this functor into a strong symmetric monoidal functor. In particular, given a commutative simplicial monoid $M$, there is a natural commutative $\Gamma$-ring $\mathbb{S}[M]=$ $\mathbb{S} \wedge M_{+}$, and an adjunction

$$
\operatorname{Map}_{\text {comm. monoids }}\left(M, R\left(S^{0}\right)\right) \cong \operatorname{Map}_{\text {comm. } \Gamma \text {-rings }}(\mathbb{S}[M], R) .
$$

In particular, if $M$ is the free commutative topological monoid $\mathbb{N}^{S}$ on a set $S$, then 
we get an adjunction

$$
\operatorname{Map}\left(S, R\left(S^{0}\right)\right) \cong \operatorname{Map}_{\text {comm. }} \text {-rings }\left(\mathbb{S}\left[\mathbb{N}^{S}\right], R\right) .
$$

For a symmetric spectrum $Y$ and a based space $K$ we also have a symmetric spectrum $Y \wedge K$, and the functor $\mathbb{S} \wedge(-)$ is strong symmetric monoidal. There is a natural isomorphism $\mathcal{S} p(X \wedge K) \cong \mathcal{S} p(X) \wedge K$.

We now briefly recall the Dyer-Lashof operations [2]. Let $R$ be a commutative symmetric ring spectrum, $H=\mathbb{H F}_{p}$ be a commutative symmetric ring spectrum modeling the mod- $p$ Eilenberg-MacLane spectrum, and $T=H \wedge R$ (although $T$ can in general be any commutative $H$-algebra). Given an element $\alpha \in H_{k}\left(R ; \mathbb{F}_{p}\right)=\pi_{k}(T)$, we can choose a fibrant replacement $T \rightarrow \tilde{T}$ and a representing map $\alpha: S^{k} \rightarrow \tilde{T}$. Taking $p$-fold smash products over $H$ and considering the natural map from homotopy orbits to orbits, we obtain a diagram of symmetric spectra as follows:

$$
\left(S^{k}\right)_{h \Sigma_{p}}^{\wedge p} \rightarrow \tilde{T}_{h \Sigma_{p}}^{\wedge_{H} p} \stackrel{\sim}{\longleftarrow} T_{h \Sigma_{p}}^{\wedge_{H} p} \rightarrow T^{\wedge_{H} p} / \Sigma_{p} \rightarrow T .
$$

Here the right-hand map is the multiplication map. By adjunction we obtain a total power operation

$$
P(\alpha): H_{*}\left(\left(S^{k}\right)_{h \Sigma_{p}}^{\wedge p} ; \mathbb{F}_{p}\right) \rightarrow H_{*}\left(R ; \mathbb{F}_{p}\right)
$$

The images of particular generators of homology on the left-hand side are the DyerLashof operations on $\alpha$. By construction these operations are natural in $R$. If $k$ is even or $p=2$, then the homology on the left-hand side is a shift of the homology of $B \Sigma_{p}$ via the Thom isomorphism.

Theorem 2.5. If $R$ is a commutative $\Gamma$-ring, then the elements of $H_{0}\left(\mathcal{S} p(R) ; \mathbb{F}_{p}\right)$ vanish under all Dyer-Lashof operations of positive degree.

Proof. Let $S \subset R\left(S^{0}\right)$ be a set of representatives for $\pi_{0} R\left(S^{0}\right)$. By Lemma 2.3 and the adjunction of equation 1 , there is a natural map $\mathbb{S}\left[\mathbb{N}^{S}\right] \rightarrow R$ of commutative $\Gamma$-rings such that the associated map

$$
\mathcal{S} p\left(\mathbb{S}\left[\mathbb{N}^{S}\right]\right) \rightarrow \mathcal{S} p(R)
$$

is surjective on $\pi_{0}$. The map on $H_{0}\left(-; \mathbb{F}_{p}\right)$ is then also surjective because the resulting spectra are connective. By naturality it suffices to show that all Dyer-Lashof operations vanish on $H_{0}\left(\mathbb{S}\left[\mathbb{N}^{S}\right] ; \mathbb{F}_{p}\right)$. However, as $\mathbb{N}^{S}$ is discrete we have

$$
H_{*}\left(\mathcal{S} p\left(\mathbb{S}\left[\mathbb{N}^{S}\right]\right)\right)=H_{*}^{\text {sing }}\left(\mathbb{N}^{S}\right)=0
$$

for $*>0$.

Corollary 2.6. The free $E_{\infty}$-algebra on $S^{0}$ cannot be realized by a commutative Г-ring.

Proof. The free $E_{\infty}$-algebra on a spectrum $X$ has the homotopy type

$$
\mathbb{P}(X)=\bigvee_{k \geqslant 0}\left(X^{\wedge k}\right)_{h \Sigma_{k}} .
$$


In particular, if $X=S^{0}$, then the homology of $\mathbb{P}\left(S^{0}\right)$ is

$$
\bigoplus_{k \geqslant 0} H_{*}\left(B \Sigma_{k} ; \mathbb{F}_{p}\right)
$$

The total power operation on the generator $\alpha$ of $H_{0}\left(B \Sigma_{1} ; \mathbb{F}_{p}\right)$ is then the map

$$
P(\alpha): H_{*}\left(B \Sigma_{p} ; \mathbb{F}_{p}\right) \rightarrow \bigoplus_{k \geqslant 0} H_{*}\left(B \Sigma_{k} ; \mathbb{F}_{p}\right),
$$

given by the inclusion of a summand, and in particular nontrivial in infinitely many positive degrees. (In fact, the right-hand side is the free unstable algebra over the Dyer-Lashof algebra on the generator $\alpha$.)

Remark 2.7. It is not clear whether there are further restrictions on the Dyer-Lashof structure of a commutative $\Gamma$-ring. For example, the Eilenberg-MacLane object $\mathbb{H F}_{2}$ can be realized by a commutative $\Gamma$-ring $H$ with

$$
H\left(X_{+}\right)=\bigoplus_{x \in X} \mathbb{F}_{2}
$$

The mod-2 homology $H_{*}\left(\mathcal{S} p(H) ; \mathbb{F}_{2}\right)$ is the dual Steenrod algebra $\mathcal{A}_{*}$, whose DyerLashof structure is elaborated upon in $[\mathbf{2}]$ and can be deduced from the Nishida relations. In particular, one can show, by applying the operation $\left(S q^{m+1}\right)_{*}$ dual to $S q^{m+1}$, that the generator $\xi_{1}$ of the dual Steenrod algebra in degree 1 supports nonzero Dyer-Lashof operations $Q^{m}$ for all $m \geqslant 1$.

A natural further question is then whether less "rigid" examples, such as the spherical group ring of a 0 -connected infinite loop space, admit models as commutative r-rings.

\section{References}

[1] A.K. Bousfield and E.M. Friedlander, Homotopy theory of $\Gamma$-spaces, spectra, and bisimplicial sets, in Geometric applications of homotopy theory (Proc. Conf., Evanston, Ill., 1977), II, Lecture Notes in Math. 658, Springer-Verlag, New York, 1978, pp. 80-130.

[2] R.R. Bruner, J.P. May, J.E. McClure and M. Steinberger, $H_{\infty}$ ring spectra and their applications, Lecture Notes in Math. 1176, Springer-Verlag, New York, 1986.

[3] A.D. Elmendorf, I. Kriz, M.A. Mandell and J.P. May, Rings, modules, and algebras in stable homotopy theory, Math. Surveys Monogr. 47, Amer. Math. Soc., Providence, RI, 1997, With an appendix by M. Cole.

[4] M. Hovey, B. Shipley and J. Smith, Symmetric spectra, J. Amer. Math. Soc. 13 (2000), no. 1, 149-208.

[5] L.G.Lewis, Jr., Is there a convenient category of spectra?, J. Pure Appl. Algebra 73 (1991), no. 3, 233-246.

[6] M. Lydakis, Smash products and $\Gamma$-spaces, Math. Proc. Cambridge Philos. Soc. 126 (1999), no. 2, 311-328. 
[7] M.A. Mandell, J.P. May, S. Schwede and B. Shipley, Model categories of diagram spectra, Proc. London Math. Soc. (3) 82 (2001), no. 2, 441-512.

[8] S. Schwede, Stable homotopical algebra and $\Gamma$-spaces, Math. Proc. Cambridge Philos. Soc. 126 (1999), no. 2, 329-356.

[9] G. Segal, Categories and cohomology theories, Topology 13 (1974), 293-312.

Tyler Lawson tlawson@math.umn.edu

Department of Mathematics, University of Minnesota, 206 Church Street S.E., Minneapolis, MN, 55414, U.S.A. 